\title{
DESIGN, FABRICATION AND TESTING OF A MEMS SYRINGE
}

\author{
Boris Stoeber, and Dorian Liepmann \\ Berkeley Sensor and Actuator Center, University of California at Berkeley \\ Berkeley, Ca 94720-1774
}

\begin{abstract}
Drugs can be delivered effectively and painlessly into the epidermal layer under the stratum corneum above the capillary bed into which the drug diffuses. This approach has even been demonstrated for lyophilized compounds that are advantageous for many situations because of their long-term stability. For this purpose a disposable syringe has been developed and fabricated using a combination of standard silicon MEMS technology and soft lithography. The MEMS syringe is based on an array of hollow pointed silicon microneedles and a deformable PDMS (polydimethylsiloxane) reservoir for a suspension of lyophilized drug. The associated phenomenon of clog formation in the needle channel due to shear-induced particle sedimentation is described in terms of its impact on system design. The complete system has been successfully tested on a model tissue.
\end{abstract}

\section{INTRODUCTION}

The need for a technology that produces disposable devices, such as MEMS, could provide an ideal platform for the widespread distribution of pharmaceuticals such as vaccines without the need for environmentally-controlled storage. MEMS technology could also provide a means to administer a pharmaceutical compound without requiring trained personnel to prepare or give the shot. A very simple concept for a disposable syringe is shown in Fig.1. A deformable reservoir on the backside of an array of microneedles contains a lyophilized drug suspended in a non-aqueous fluid. Merely pressing the device against the skin delivers the medicine or vaccine. Such a device would be ideal for wide-spread distribution of vaccines in third-world situations where the lack of storage and trained personnel are significant problems.

Many therapeutic agents including most antibiotics are unstable in aqueous solution, which is the usual formulation for delivery. Either the medication has to be prepared just before use or the prepared formulation has to be kept cool to prolong its efficacy. Dehydrated or lyophilized compounds are significantly safer. In addition, lyophilized medications have been shown to be bio-available when injected between the stratum corneum and the capillary bed. An additional advantage is that the injection will be painless at these depths $(20-100 \mathrm{ìm})$ in the epidermis because the needles do not reach the upper nerve cells. The drug, however, is able to diffuse deeper into the skin and is absorbed into the capillaries of the dermis.

Microneedles have been an active area of research for almost a decade [1-11] for biomedical applications including $\mu$ TAS (micro Total Analysis Systems), yet integration of such needles into complete microsystems has not been performed mainly because of process compatibility problems. The device presented here uses two different materials, silicon and PDMS, that can be photolithographically defined to be the micro-needle array and the drug reservoir.

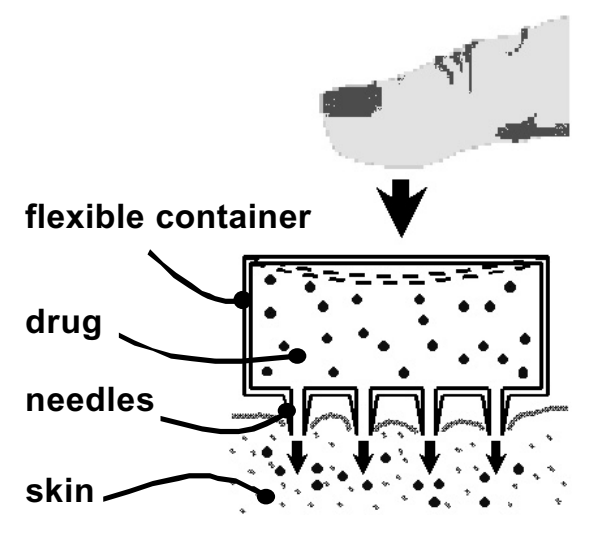

Figure 1. Concept of a disposable MEMS syringe.

\section{NEEDLE DESIGN AND FABRICATION}

Needles fabricated using MEMS technology can be divided into two major groups; in-plane needles where the needle shaft is fabricated in a plane parallel to the substrate [1-5], and out-ofplane needles, which have their shafts perpendicular to the wafer plane [6-11]. Some of the in-plane needle designs allow for integration of electronics, but they can only be arranged in single rows, and mainly problems associated with interconnects have prevented them from being integrated into complete micro systems. The major advantage of out-of-plane needles is the possibility to arrange them in arrays so that fluid can be delivered or sampled over a wider area making the system more stable and robust [9]. These considerations make arrays of hollow pointed out-of plane needle the most adaptable for a MEMS syringe, since these needles are rigid, easily penetrate the skin and have multiple orifices so that the syringe cannot be plugged unexpectedly.

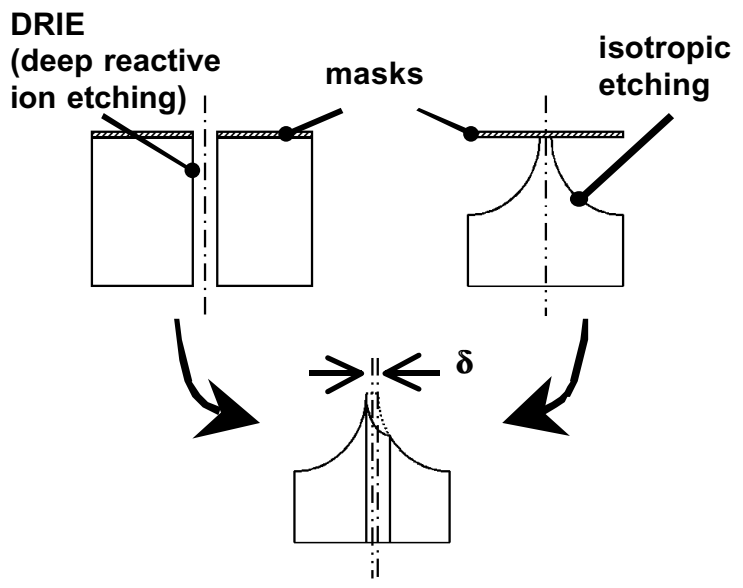

Figure 2. Design of a pointed hollow out-of-plane needle.

Travel support has been generously provided by the Transducers Research Foundation and by the DARPA MEMS and DARPA BioFlips programs. Research support by Becton-Dickinson and the DARPA Simbiosys program is also gratefully acknowledged. Simulations were performed using CFD ACE+ software provided by CFD Research Corporation, Huntsville, AL. 
The design shown in Fig.2 has been fabricated using the Bosch process [12] for DRIE (deep reactive ion etching) to etch the needle channel through the substrate, while the outer shape of the needle is obtained through under-etching of a circular mask with isotropic etching techniques while the sidewalls of the needle channel is protected by a passivation layer. An offset $\delta$ of the center lines of the two corresponding etch masks results in a needle with a sharp tip on the circumference of its shaft.

Figure 3 shows an electron micrograph of such an array of needles. The design permits a density of 600 needles $/ \mathrm{cm}^{2}$ for shaft lengths of $200 \mu \mathrm{m}$. Figure 4 allows size comparison of such silicon needles with a conventional 26 gauge stainless steel needle.

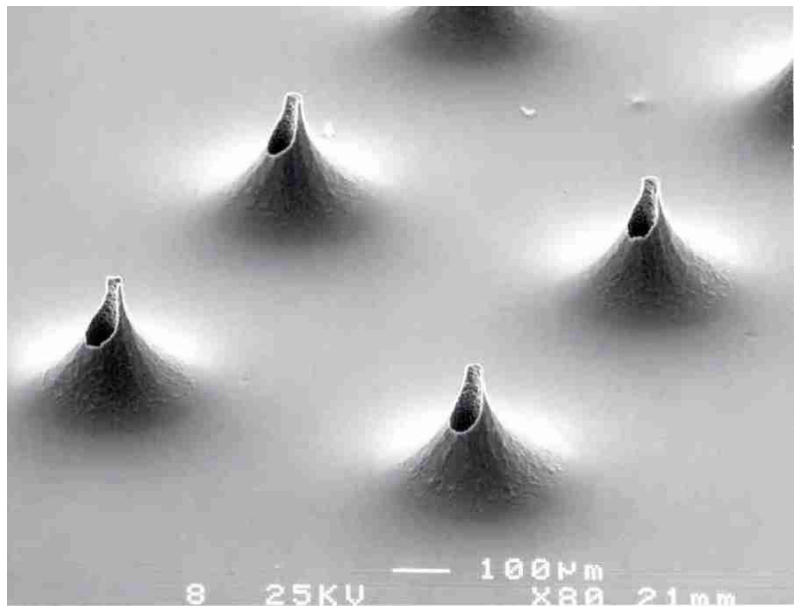

Figure 3. Array of pointed hollow out-of-plane silicon needles with height of $200 \mathrm{im}$ and channel diameter of $40 \mathrm{im}$.

\section{6 gauge stainless steel needle}

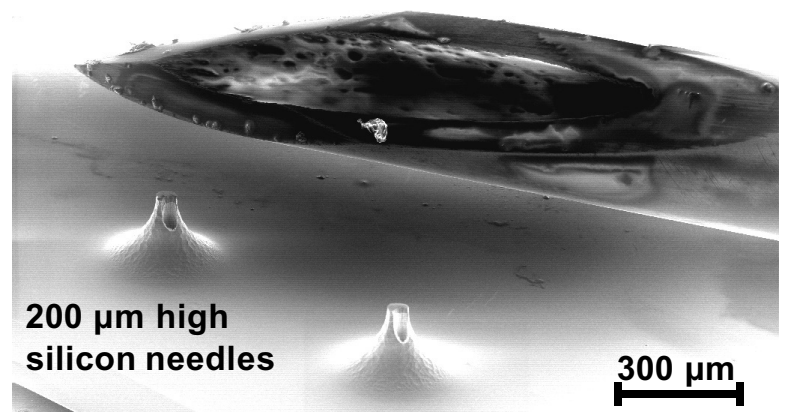

Figure 4. Size comparison of a 26 gauge stainless steel needle and an early version of silicon needles.

\section{FLUID MECHANICAL DESIGN}

The nature of suspension flow into contractions in the flow field sets an important design criterion for a MEMS syringe because we anticipate its use with a lyophilized drug. A model suspension of $0.7 \mu \mathrm{m}$ large polystyrene beads in water have been shown to agglomerate at the inlet region of a $D=50 \mu \mathrm{m}$ wide flow channel at surprisingly low flow rates $q=0.1 \mu \mathrm{l} / \mathrm{min}$ and particle concentrations $\Phi=0.1 \%$ as shown in Fig. 5 . In a short time the channel inlet completely clogs as shown in Fig.6 for 2-D suspension flow into a contraction. For any drug delivery system it is critical that the needle channnels stay open until the end of the injection process.

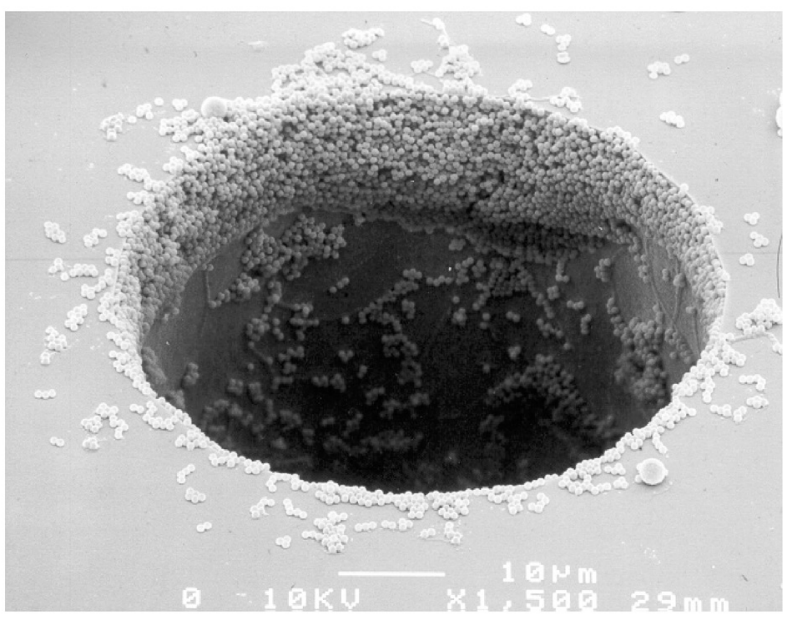

Figure 5. 0.7 $\mu \mathrm{m}$ large polystyrene beads start to agglomerate at the inlet of the flow channel.

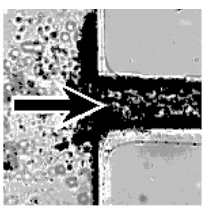

$\mathrm{t}=0 \mathrm{~min}$

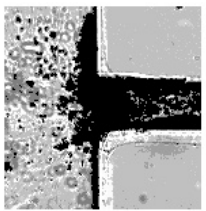

$\mathrm{t}=3 \mathrm{~min}$

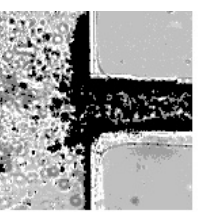

$\mathrm{t}=1 \mathrm{~min}$

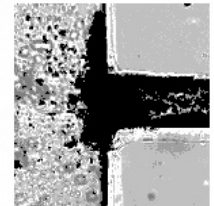

$\mathrm{t}=4 \mathrm{~min}$

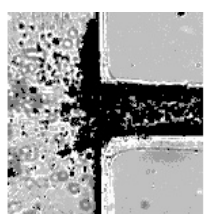

$\mathrm{t}=2 \mathrm{~min}$
Figure 6. Sequential video images of polystyrene beads $(0.92 \mu \mathrm{m})$ agglomerating and finally blocking the inlet of a 2-D channel. The particles appear as dark shadows.

Recent results based on the dimensional analysis in [13] show that the critical nondimensional number for this phenomenon

$$
\Pi=\frac{q \cdot d^{3} \cdot \tau \cdot \Phi}{D^{6}}
$$

can be used to determine a typical time scale $\tau$ for clog formation for suspension flow into a flow channel. The most critical parameter of this fluid mechanical problem is the diameter of the flow channel $D$, related to Eq.(1) through the size parameter $D / d$ to the third power and through the nondimensional shear rate [13] $q \tau / D^{3}$. In the case where this time scale needs to be reduced and the size of the particles $d$ is fixed the most effective adjustment can be achieved by increasing the channel diameter. However, increasing the channel diameter reduces the sharpness of the needle tip and widens the opening along the needle shaft that could result in leakage during injection. Instead of widening the entire channel, an easily fabricated two-step channel inlet can reduce the overall shear rate in the flow as shown in Fig. 7 if appropriate scaling is maintained. If the difference in diameters for these two steps is too large, the shear rate at the second contraction is identical to the shear rate in the case of a simple contraction, so that the same typical timescale for clogging can be expected. These simulations were performed using CFD ACE+ software from CFD Research Corporation. 


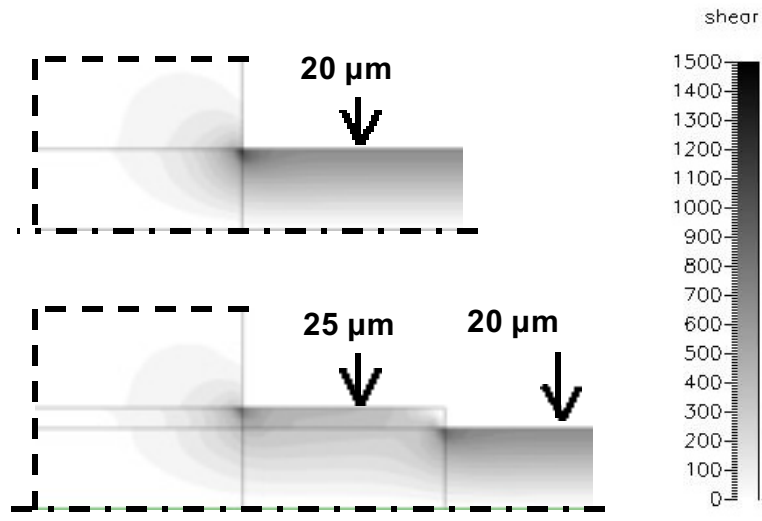

Figure 7. Computational results showing the reduction of the maximum shear rate [1/sec] for a fluid flowing into a $40 \mu \mathrm{m}$ wide channel at a flow rate of $2 \mu \mathrm{l} / \mathrm{min}$.

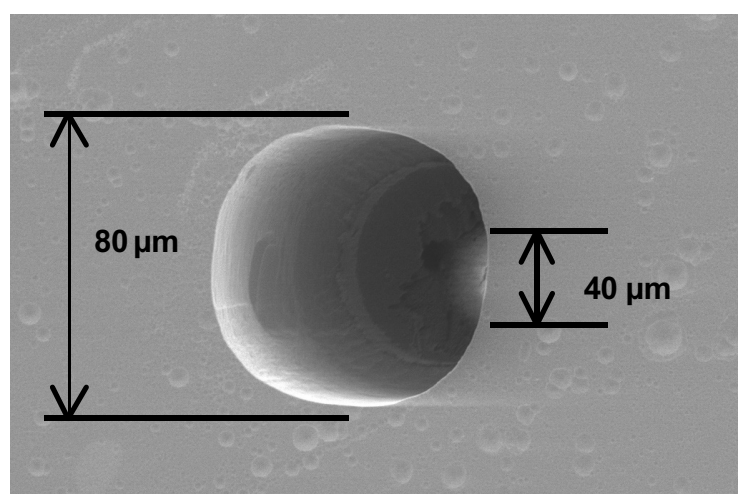

Figure 8. 2-Step channel inlet.

\section{CONTAINER FABRICATION AND INTEGRATION}

The flexible container for the drug suspension has been fabricated out of PDMS that is chemically bonded to silicon as outlined in Fig.9. Coating a silicon mold with a monolayer of HMDS facilitates the removal of the PDMS from this mold after the casting and hardening steps as shown in Fig.10. The surface of the PDMS is then activated in a low power oxygen plasma, so that contact with the backside of the silicon micro-needle array chemically bonds these two devices together [14]. This process is suitable for batch fabrication of MEMS syringes because an entire wafer size PDMS sheet can be bonded to the backside of a silicon wafer. The PDMS can then be cut along silicon dye cleavage lines with a sharp blade, while these cleavage lines need to be cut into the wafer backside prior to the isotropic etching step.

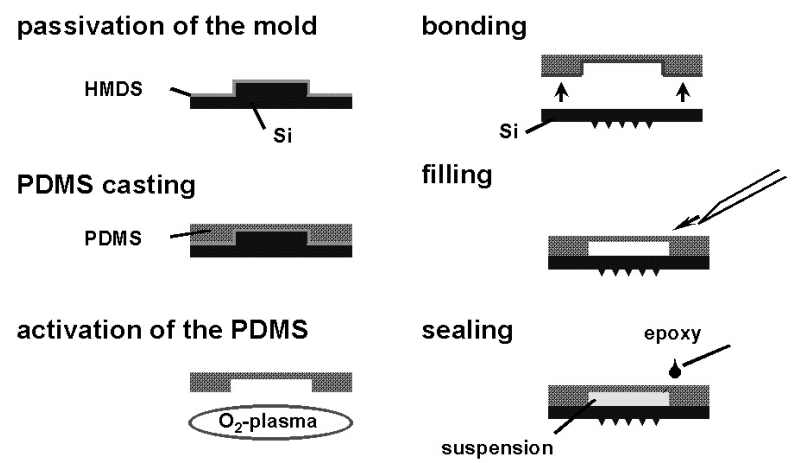

Figure 9. Process flow for reservoir fabrication and device integration.

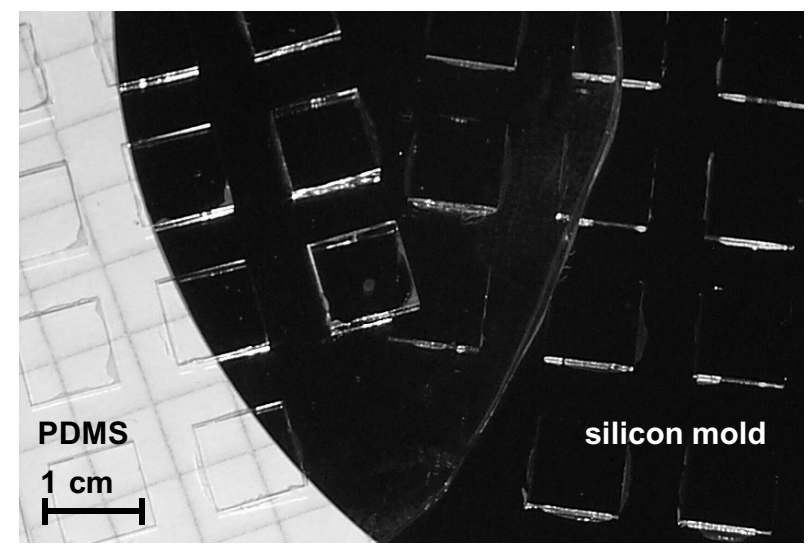

Figure 10. PDMS sheet with container grooves peeled off its silicon mold.

The PDMS container of the assembled MEMS syringe shown in Fig. 11 has been filled with a suspension of blue polystyrene microbeads in water. Its 8 needles are spaced $1 \mathrm{~mm}$ apart from each other, however much larger and denser arrays of needles are possible. The PDMS container volume of $20 \mu \mathrm{l}$ can be easily varied using different silicon molds.

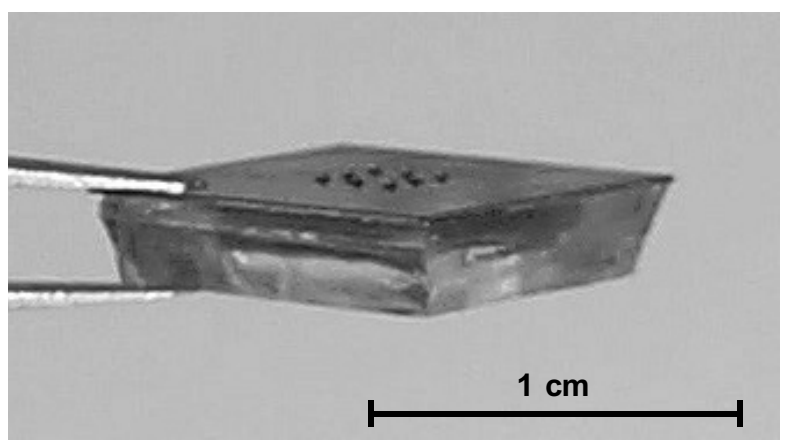

Figure 11. A MEMS syringe with 8 microneedles and a PDMS container filled with a suspension of blue microbeads.

\section{TESTING OF THE MEMS SYRINGE}

Aqueous suspensions of blue and fluorescent polystyrene beads were injected using a MEMS syringe into chicken breast as a model tissue. The surface of chicken flesh in Fig. 12 shows the 8 injection marks of the $2 \times 4$ needle array of the syringe.

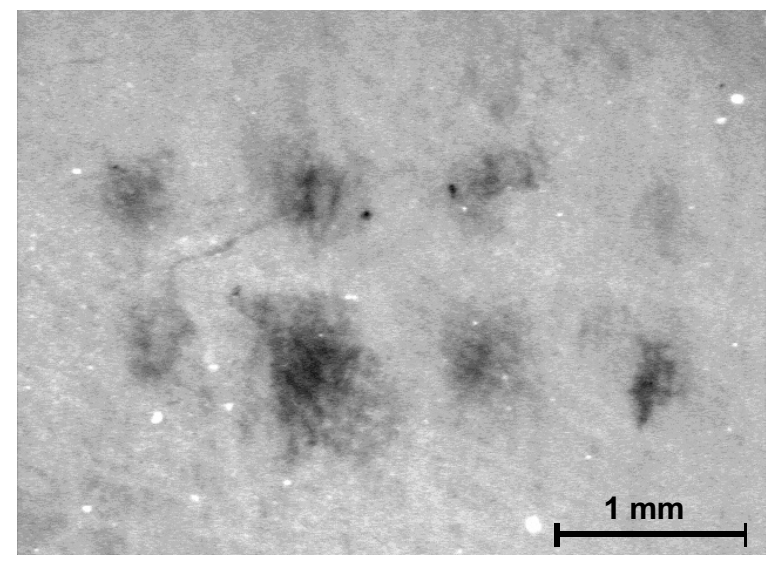

Figure 12. Injection marks of a MEMS syringe with an array of $2 \times 4$ needles on the surface of chicken breast. 
The delivery depths of fluorescent particles have been investigated using confocal microscopy (Fig.13). These results indicate that the maximum particle concentration occurred about $20 \mu \mathrm{m}$ under the surface, while a strong signal could still be detected at $70 \mu \mathrm{m}$.

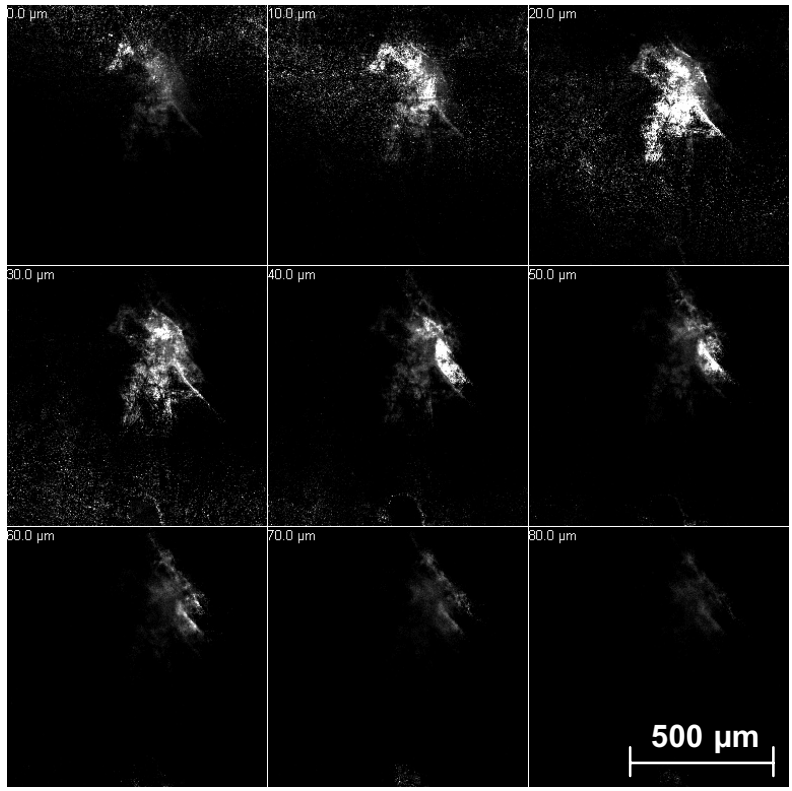

Figure 13. Confocal microscopic images of $0.7 \mu \mathrm{m}$ large fluorescent polystyrene beads at different depths in chicken tissue.

Thus the injection of a suspension of microscale particles with a MEMS syringe was successfully delivered into a model tissue at the target depth.

\section{CONCLUSIONS}

A disposable syringe has been fabricated in MEMS technology, and it has been successfully tested for injection of a sample suspension into a model tissue. The micro-particles were delivered at an appropriate depth for human skin, below the stratum corneum and above the nerves, which exist deeper in the dermal layer. Design solutions have been proposed to avoid the possibility of clog formation in the needle channels due to shear induced sedimentation of the solid phase of a drug suspension.

These results indicate that the MEMS syringe is a very promising and potentially very useful biomedical tool. However, further experiments to directly monitor drug absorption by the body would be necessary for a final validation of this drug delivery concept. In addition to delivery efficiency of the device, it will also be critical to determine how much of drug in the reservoir is delivered and its reproducibility accounting for person-to-person variability. For this reason we see this device as a tool for vaccines and antibiotics where over-dosing is not a significant problem.

\section{REFERENCES}

1. L. Lin, A.P. Pisano, and R.S. Muller, "Silicon Processed Microneedles", Proceedings ot the $7^{\text {th }}$ International Conference on Solid-State Sensors and Actuators (Transducers'93), Yokohama, Japan , 06/07-10/93, Institute of Electrical Engineers of Japan, Tokyo (1993), pp. 237-240.

2. J. Chen, K.D. Wise, "A Multichannel Neural Probe for Selective Chemical Delivery at the Cellular Level”, ", Technical Digest of the 1994 Solid-State Sensor and Actuator Workshop,
Hilton Head Isl., SC, 06/13-16/94, Transducer Research Foundation, Cleveland (1994), pp. 256-259.

3. N.H. Talbot, A.P. Pisano, "Polymolding: Two Wafer Polysilicon Micromolding of closed Flow Passages for Microneedles and Microfluidic Devices", Technical Digest of the 1998 Solid-State Sensor and Actuator Workshop, Hilton Head Isl., SC, 06/8-11/98, Transducer Research Foundation, Cleveland (1998), pp. 265-268.

4. K.S. Lebouitz, and A.P. Pisano, "Microneedles and Microlancets fabricated using SOI wafers and isotropic etching",

Proceedings of the Symposium on Microstructures and Microfabricated Systems IV, Boston, MA, 11/1-6/98, Electrochemical Society, Pennington, NJ (1998), pp. 235-244.

5. J. Brazzle, D. Bartholomeusz, R. Davies, J. Andrade, R.A. Van Wagenen, and A.B. Frazier, "Active Microneedles with Integrated Functionality", Technical Digest of the 2000 Solid-State Sensor and Actuator Workshop, Hilton Head Isl., SC, 06/04-08/00, Transducer Research Foundation, Cleveland (2000), pp. 199-202.

6. S. Henry, D.V. McAllister, M.G. Allen, and M.R. Prausnitz., "Microfabricated Microneedles: A Novel Approach to Transdermal Drug Delivery”, Journal of Pharmaceutical Sciences, 87, 8, pp. 922-925, (1998).

7. D.V. McAllister, F. Cros, S.P Davis, L.M. Matta, M.R. Prausnitz, and M.G. Allen, "Tree-Dimensional Hollow Microneedle and Microtube Arrays", Proceedings of the $10^{\text {th }}$ International Conference on Solid-State Sensors and Actuators (Transducers'99), Sendai, Japan 06/7-10/99, Institute of Electrical Engineers of Japan, Tokyo (1999), pp. 1098-1101.

8. K. Chun, G. Hshiguchi, H. Toshiyoshi, H.Fujita, Y. Kikuchi, J. Ishikawa, Y. Murakami, and E. Tamiya, "An Array of Hollow Microcapillaries for the Controlled Injection of Genetic Materials into Animal/Plant Cells", Proceedings of $12^{\text {th }}$ International Workshop on Micro Electro Mechanical Systems, Orlando, FL, 01/17-21/99, IEEE, Piscataway (1999), pp. 406-412.

9. B. Stoeber, and D. Liepmann, "Fluid Injection Through OutOf-Plane Microneedles", Proceedings of $1^{\text {st }}$ Annual International IEEE-EMBS Special Topic Conference on Microtechnologies in Medicine and Biology, Lyon, France, 10/12-14/00, IEEE, Piscataway (2000), pp. 224-228.

10. J.G.E. Gardeniers, J.W. Berenschot, M.J. de Boer, Y. Yeshurun, M. Hefetz, R. van 't Oever, and A. van den Berg, "Silicon Micromachined Hollow Microneedles for Transdermal Liquid Transfer", Proceedings of $15^{\text {th }}$ International Conference on Micro Electro Mechanical Systems, Las Vegas, NV, 01/20-24/02, IEEE, Piscataway (2002), pp. 141-144.

11. P. Griss, G. Stemme, "Novel, Side Opened Out-of-Plane Microneedles for Microfluidic Transdermal Interfacing”,

Proceedings of $15^{\text {th }}$ International Conference on Micro Electro Mechanical Systems, Las Vegas, NV, 01/20-24/02, IEEE, Piscataway (2002), pp. 467-470.

12. F. Lärmer, and P. Schilp, German Patent DE 4241045 (1994).

13. B. Stoeber, and D. Liepmann, "Operational Limits of Suspension Flow through Sudden Contractions", Proceedings of the 2001 ASME international Mechanical Engineering Congress and Exposition, New York, NY, 11/11-16/01, ASME (2001), disc set vol.2, IMECE2001/MEMS-23878.

14. D.C. Duffy, J.C. McDonald, O.J.A. Schueller, and G.M. Whitesides, "Rapid Prototyping of Microfluidic Systems in Poly(dimethylsiloxane)", Anal. Chem, 70, pp. 4974-4984 (1998). 